\title{
Microcytic and Malarial Anaemia Prevalence in Urban Children $\leq 15$ Years in the Mount Cameroon Area: A Cross-Sectional Study on Risk Factors
}

\author{
Sharon Odmia Sama, ${ }^{1}$ Seraphine Njuontsop Chiamo, ${ }^{1}$ Germain Sotoing Taiwe, ${ }^{1}$ \\ Gwendolyne Elobe Njume, ${ }^{1}$ and Irene Ule Ngole Sumbele $\mathbb{i}^{1,2}$ \\ ${ }^{1}$ Department of Zoology and Animal Physiology, University of Buea, Buea, Cameroon \\ ${ }^{2}$ Department of Microbiology and Immunology, Cornell College of Veterinary Medicine, Ithaca, NY, USA \\ Correspondence should be addressed to Irene Ule Ngole Sumbele; sumbelei@yahoo.co.uk
}

Received 7 July 2020; Revised 22 November 2020; Accepted 30 March 2021; Published 8 April 2021

Academic Editor: Duran Canatan

Copyright (C) 2021 Sharon Odmia Sama et al. This is an open access article distributed under the Creative Commons Attribution License, which permits unrestricted use, distribution, and reproduction in any medium, provided the original work is properly cited.

\begin{abstract}
Background. Anaemia, a common nutritional deficiency, is a public health problem in the Mount Cameroon area. This study determined the prevalence and possible risk factors of microcytic and malarial anaemia in children less than $\leq 15$ years residing in the Buea and Limbe municipalities in the Mount Cameroon area. Methods. A total of 566 children were clinically examined in a cross-sectional study from December 2018 to August 2019 for anaemia and malaria parasites. Blood samples collected were used in evaluating full blood count with the aid of an automated haemoanalyser, and malaria parasite was confirmed by microscopy. Anaemia was defined based on WHO standards while microcytic anaemia and malarial anaemia were defined as microcytosis + anaemia and malaria + anaemia, respectively. Factors that showed significance in the bivariate analysis were entered into a multinomial logistic regression to determine risk factors for microcytic and malarial anaemia. Results. The overall prevalence for anaemia, microcytosis, microcytic anaemia, and malarial anaemia was, respectively, $68.7 \%, 48.9 \%, 36.9 \%$, and $19.6 \%$ with microcytic anaemia representing $53.7 \%$ of all anaemic cases. Risk factors for microcytic anaemia included child age of $1-5$ years $(P=0.007)$, forest ethnicity $(P=0.019)$, parents being farmers $(P=0.038)$ or jobless $(P=0.009)$, and having moderate malaria parasitaemia $(P=0.048)$ while those for malarial anaemia were child age of $6-10$ years $(P=0.008)$, parents' age of 26-35 years $(P=0.049)$, parents being jobless $(P=0.023)$, and consuming plantains $3-4$ times $(P=0.024)$ a week. Conclusion. Microcytic anaemia is getting to be a severe public health concern while malarial anaemia is a mild public health issue in children residing in urban areas of Mount Cameroon. Parents' occupation was directly linked to all anaemia forms; hence, any intervention to curb anaemia should consider aspects that will raise the socioeconomic status of the population.
\end{abstract}

\section{Introduction}

Anaemia is still a public health problem in developing countries [1] across all age groups [2]. It is a condition where blood haemoglobin concentration is lower than the normal for a person's age, gender, and environment $[3,4]$. Notably pregnant women, and children because of rapid development and impact on cognitive development, are most at risk of anaemia. The adverse health consequences and impact are felt at both the individual and societal levels [5-8]. Globally, anaemia prevalence stands at around 2 billion with over 800 million anaemic children and women; Africa and Asia bear the brunt with an estimated $85 \%$ prevalence [5]. In Cameroon, as in the Mount Cameroon area, anaemia is still a severe public health problem. Most recent estimates from the World Bank show a prevalence of $62.5 \%$ in children under five and $49.3 \%$ in pregnant women [9].

Anaemia is not a standalone condition but results from lack of one or more micronutrients, infections such as malaria and hookworm, congenital haemolytic diseases, poor maternal health, socioeconomic status, and even demographic factors [10-12]. Malaria is often associated 
with anaemia and is a major contributor to the occurrence and severity of anaemia in malaria endemic zones. Its route of action is seen in the destruction of both parasitized and nonparasitized red cells [13]. The parasites feed on host haemoglobin, and to stop them from acquiring host iron, the recycling of iron by reticuloendothelial (RE) macrophages is impeded $[14,15]$. This leads to lack of iron in the cell that may ultimately lead to iron deficiency (ID) and consequently microcytic red cells and anaemia.

Microcytic anaemia which is characterised by smaller than normal circulating red blood cell for age [16] is a common type of anaemia. While iron deficiency is the main cause of microcytic anaemia, thalassaemia trait (which is a defect in haemoglobin synthesis), chronic inflammation (resulting from lack of iron), and other haemoglobinopathies (such as sideroblastic anaemia) are implicated [17]. Microcytic anaemia often goes undetected except incidentally encountered when full blood count is run for other reasons [18], hence, the need for regular monitoring of its occurrence. Another anaemia type of common occurrence is iron deficiency anaemia (IDA) which results from depleted iron stores due to poor absorption or poor or reduced iron intake and poor erythropoiesis [19]. However, a person can be functionally iron deficient without out-rightly manifesting anaemia as often seen in mild to severe anaemia. Nonetheless, as IDA worsens, the red cells of its victims are exposed to oxidation and reduced antioxidant defence [20].

While several studies in the Mount Cameroon area have determined the occurrence of anaemia in conjunction with malaria and other infections in various setting, the type of anaemia which is invaluable in making informed decision when planning control measures has been infrequently examined. Therefore, the objective of this study was to determine the prevalence and identify factors associated with microcytic and malarial anaemia in children $\leq 15$ years living in urban areas of Buea and Limbe in the Mount Cameroon area.

\section{Methods}

2.1. Study Sites and Participants. The study was conducted in the Buea and Limbe I and III municipalities, both located in the Mount Cameroon area of Fako Division. Buea is situated and characterised as described by previous studies [21]. It is divided into 7 health areas, namely, Bokwango, Bova, Buea Road, Buea Town, Molyko, Muea, and Tole. Limbe has been described by previous studies [22]. In terms of health areas, the Limbe Health District is subdivided further into 8 health areas, namely, Batoke, Bimbia, Bojongo, Bota, Mabeta, Moliwe, Limbe Regional Hospital, and Sanje. These 8 health areas fall under 3 councils: Limbe I (Poh), Limbe II, and Limbe III councils. Malaria parasite transmission in Cameroon is very heterogenous varying with altitude and climate [23]. In the Mount Cameroon area, transmission is holoendemic and perennial with lower altitudes having the highest transmission rates [24]. Entomological inoculation rates (EIR) reported in the area before vector control measures were implemented ranged from 149.0 to 287.0 infectious bites per person per year (ib/p/y) in coastal towns like Limbe,
Tiko, and Idenau [25]. This rate witnessed a drop to $0.7-1.4 \mathrm{ib} /$ $\mathrm{p} / \mathrm{m}$ [26] after the use of long-lasting insecticide-treated bed nets (LLINs) was implemented.

The study population included children $1-15$ years living in the study area whose parents or caregivers gave consent to their participation in the study. Those with known HIV status, with active haemorrhage, who had blood transfusion or were operated upon two months prior to the study, and who are or had been on antimalarials two weeks prior to the study were excluded.

2.2. Study Design, Sampling Technique, and Unit. This crosssectional study was carried out from December 2018 to August 2019. Following administrative and ethical clearances, participants were enrolled into the study at their various communities and at presentation to the hospital following education. Informed consent/assent forms were given to parents/caregivers explaining the purpose, benefits, and risks of the study. Structured questionnaires to obtain information on sociodemographic data, type of accommodation, clinical history, and dietary habits were administered, and clinical evaluation and blood samples were collected thereafter for malaria parasite determination and full blood count (FBC) analyses.

A convenient multistage sampling method was employed in the study. Firstly, for representativeness of each health district, a health area was randomly selected from each of the 3 council areas that make up the Limbe Health District and 2 were selected from the 4 distinct urban settings recognised in the Buea Health District. This was followed by a random selection of representative health facilities and neighbourhoods in the selected health areas. Following education by the community relay agents, potential participants in the selected communities were invited to a specified data collection hall in the neighbourhood on programmed dates coordinated by the neighbourhood head. Concurrently, at presentation to the outpatient department of both Buea Regional and Bota District Hospitals, participants were enrolled prospectively as they fulfilled the inclusion criteria for the study. The sample size for the study was determined using the formula $n=z^{2} p q / d^{2}$ [27], where $n$ was the required sample size; $z$ was 1.96 , the standard deviation for a $95 \%$ confidence interval (CI); $P$ value was $62 \%$ which was the anaemia prevalence in the region [21]; $q$ was $(1-p)$; and $d$ was the acceptable error margin set at 0.05 . The optimum sample size for both health districts was 362 . To allow for data loss due to incomplete data entry, the sample size was increased by $10 \%$ to 400 requiring an average of 200 samples per health district.

2.3. Data Collection. Each child was examined clinically by a trained physician. With the assistance of an interviewer, the parent/caregiver was given a self-administered structured questionnaire to fill, and this included information on sociodemography, clinical symptoms, type of habitation, and dietary habits. Axillary temperature was measured using a clinical thermometer, and fever was defined as temperature $\geq 37.5^{\circ} \mathrm{C}$. Symptoms such as cough, headache, diarrhoea, and body weakness/pain were recorded. Weight and height were 
measured using a Terraillon weighing scale and a measuring tape to the nearest $0.1 \mathrm{~kg}$ and $1 \mathrm{~cm}$, respectively. Parents of children who could not walk were requested to climb on the balance with the child and then without the child; the difference between both weights was recorded as the child's weight. The recumbent length was measured for children who could not walk. Height-for-age (HA), weight-for-age (WA), and weight-for-height (WH) z-scores were computed based on WHO growth curves [28] using WHO Anthro and AnthroPlus packages [29]. Stunting, underweight, and wasting were, respectively, defined as $\mathrm{HA}$, WA, and $\mathrm{WH} z$ scores of $<-2$. Malnutrition was defined as any $z$-score $<-2$ [16]. Dietary assessment was done by a recall by parents on how often in a week they consumed the following foods: fruits, vegetables, meat, fish, and plantains. This was scored as (1) 1-2 times, (2) 3-4 times, and (3) $>4$ times a week.

2.4. Laboratory Methods. Approximately $4 \mathrm{~mL}$ of venous blood was collected using sterile techniques into EDTA and dry tubes. Labelled blood samples were transported on ice to the Malaria Research Laboratory of the University of Buea for further analysis. Thin and thick blood films were prepared immediately after dispensing blood into EDTA tubes. Thin films were fixed with absolute methanol and together with the thick films stained for 15 minutes with $10 \%$ Giemsa stain and then examined for the detection and identification of the malaria parasite following standard procedures [30]. Slides were considered positive when asexual/gametocyte forms were observed, and parasitaemia was calculated per 200 white cells multiplied by patient's white blood cell (WBC) counts and stretched to 500 leukocytes if gametocytes were seen [30]. Parasite burden was classified per microliter $(\mu \mathrm{L})$ of blood as low ( $<1000$ parasites), moderate (1000-4999 parasites), high (5000-99,999 parasites), and hyper ( $\geq 100,000$ parasites) [31].

Blood in the dry tube was centrifuged at $3000 \mathrm{rpm}$ for 5 minutes, and the aliquots were stored at $-20^{\circ} \mathrm{C}$ until use. C-reactive protein (CRP) concentration was determined using the Thermo Fisher ELISA machine as per the manufacturer's instructions. Inflammation was confirmed when acute phase protein (APP) CRP was $>5 \mathrm{mg} / \mathrm{L}$ [20]. A complete blood count was run using the Nihon Kohden Celltac $\alpha$ haemoanalyser according to manufacturer's instructions to obtain values for WBC, red blood cell (RBC) and platelet (Plt) counts, haemoglobin $(\mathrm{Hb})$, haematocrit $(\mathrm{Hct})$, mean cell volume (MCV), mean cell haemoglobin $(\mathrm{MCH})$, mean cell haemoglobin concentration (MCHC), red cell distribution width coefficient of variation (RDW-CV), red cell distribution width standard deviation (RDW-SD), mean platelet volume (MPV), and platelet distribution width (PDW). Anaemia was classified based on WHO age-based classification [32] as $\mathrm{Hb}<11.0 \mathrm{~g} / \mathrm{L}$ for children $1-5$ years, $\mathrm{Hb}<11.5 \mathrm{~g} / \mathrm{dL}$ for children $6-11$ years, and $\mathrm{Hb}<12.0 \mathrm{~g} / \mathrm{dL}$ for children $12-15$ years old. Severe anaemia was defined as $\mathrm{Hb}<7.0 \mathrm{~g} / \mathrm{dL}$ for all children. Moderate anaemia was defined as $\mathrm{Hb}: 7.0-9.9 \mathrm{~g} / \mathrm{dL}$ for $1-5$ years and $\mathrm{Hb}: 7.0-10.9 \mathrm{~g} / \mathrm{dL}$ for children 6-15 years, and mild anaemia was defined as $\mathrm{Hb}: 10.0-10.9 \mathrm{~g} / \mathrm{dL}$ for children $1-5$ years, Hb: $11.0-11.4 \mathrm{~g} /$
$\mathrm{dL}$ for children 6-11 years, and $\mathrm{Hb}: 11.0-11.9 \mathrm{~g} / \mathrm{dL}$ for children $12-15$ years. Malaria anaemia (MA) was defined as anaemia + positive blood smear for malaria parasite while nonmalarial anaemia (NMA) was defined as anaemia with negative blood smear for malaria parasites. Microcytosis was defined as MCV $<67 \mathrm{fL}$ for children less than 2 years and $\mathrm{MCV}<73 \mathrm{fL}$ for children 2 to 15 years, and microcytic anaemia was defined as $\mathrm{Hb}<11.0 \mathrm{~g} / \mathrm{dL}+\mathrm{MCV}<67 \mathrm{fL}$ and $\mathrm{Hb}<11.0 \mathrm{~g} / \mathrm{dL}+\mathrm{MCV}<73 \mathrm{fL}$, respectively [16].

2.5. Data Analysis. Data was keyed into Microsoft Excel 2010 and exported into IBM-Statistical Package for Social Sciences (SPSS) version 20 (SPSS, Inc., Chicago, IL, USA). Continuous variables were summarized as means and standard deviations while categorical variables were reported as percentages and frequencies. Proportion difference was evaluated using Pearson's Chi square test $\left(\chi^{2}\right)$, Pearson's ranked correlation $(r)$ for haematological bivariate association, and analysis of variance (ANOVA) to compare group means. Parasite density was log-transformed before analysis. Factors with a $P$ value $<0.2$ in the bivariate analysis were entered into a multinomial logistic regression to determine risk factors for microcytic and malarial anaemia. Odd ratios (OR) and 95\% confidence intervals (CIs) were computed, and $P<0.05$ value was suggestive of statistical significance.

2.6. Ethical Consideration. Ethical clearance was obtained from the Institutional Review Board hosted by the Faculty of Health Sciences, University of Buea (2018/811-05/UB/SG/ IRB/FHS), following an administrative approval from the Regional Delegation of Public Health (R11/MINSANTE/ SWR/RDPH/PS/430/940). Authorisations from community heads were also obtained. Only children whose parents consented to their participation in the study and who responded to the questionnaires were enrolled after the purpose, risks, and benefits were clearly explained to them. It was stressed that the study was completely voluntary and that a parent had every right to stop/withdraw their child from the study. Even after the parent's consent to participate in the study, any child who was too scared of a needle was not forced to continue. Data was treated with utmost confidentiality by assigning codes to the patients' samples.

\section{Results}

3.1. Baseline Characteristics of Study Participants. A total of 614 participants were approached to participate in the study, and 589 of them were enrolled. After data curation, 566 participants were retained of which $47.2 \%$ were males and $52.8 \%$ were females, living in low (61.5\%) and highland $(38.5 \%)$ areas. The mean (SD) age was $6.4(4.5)$ years of whom $51.4 \%$ were $\leq 5$ years. As shown in Table 1 , most of the participants were enrolled at presentation to hospital (63.3\%), had household head between 35 and 50 years old (44.2\%), and had secondary level of education (40.6\%). Overall, anaemia, malaria, microcytosis, inflammation, fever, and malnutrition prevalence in the study population 
TABle 1: Characteristics of the 566 participants enrolled in the study.

\begin{tabular}{|c|c|}
\hline Parameter & Total \\
\hline$\%(n)$ & $100(566)$ \\
\hline \multicolumn{2}{|l|}{ Gender } \\
\hline Female & $52.8(299)$ \\
\hline Male & $47.2(267)$ \\
\hline \multicolumn{2}{|l|}{ Age group in years } \\
\hline $1-5$ & $51.4(291)$ \\
\hline $6-10$ & $24.9(141)$ \\
\hline $11-15$ & $23.7(134)$ \\
\hline Mean age (SD) in years & $6.4(4.5)$ \\
\hline Mean height $(\mathrm{SD})$ in $\mathrm{cm}$ & $112.6(28.4)$ \\
\hline Mean weight (SD) in $\mathrm{kg}$ & $23.7(14.6)$ \\
\hline Mean $\mathrm{Hb}$ conc. in $\mathrm{g} / \mathrm{dL}$ & $10.4(2.0)$ \\
\hline \multicolumn{2}{|l|}{ Sociodemographic factors } \\
\hline \multicolumn{2}{|l|}{ Altitude of residence } \\
\hline Lowland & $61.5(348)$ \\
\hline Highland & $38.5(218)$ \\
\hline \multicolumn{2}{|l|}{ Point of presentation } \\
\hline Community & $36.7(208)$ \\
\hline Hospital & $63.3(358)$ \\
\hline \multicolumn{2}{|l|}{ Health district } \\
\hline Buea & $37.5(212)$ \\
\hline Limbe & $62.5(354)$ \\
\hline Age of household head (years) ${ }^{a}$ & $N=407$ \\
\hline$\leq 25$ & $3.9(16)$ \\
\hline $26-35$ & $28.5(116)$ \\
\hline $36-50$ & $44.2(180)$ \\
\hline$>50$ & $23.3(95)$ \\
\hline \multicolumn{2}{|l|}{ Education level of household head } \\
\hline Primary & $23.3(132)$ \\
\hline Secondary & $40.6(230)$ \\
\hline Tertiary & $31.6(179)$ \\
\hline No formal & $4.4(25)$ \\
\hline \multicolumn{2}{|l|}{ Family size } \\
\hline$\leq 5$ & $50.5(286)$ \\
\hline $6-10$ & $43.8(248)$ \\
\hline$>10$ & $5.7(32)$ \\
\hline \multicolumn{2}{|l|}{ Clinical factors } \\
\hline Mean temperature $(\mathrm{SD})$ in ${ }^{\circ} \mathrm{C}$ & $37.1(1.1)$ \\
\hline Malaria parasite prevalence & $27.7(157)$ \\
\hline Fever prevalence & $27.7(157)$ \\
\hline Prevalence of inflammation & $58.4(211)$ \\
\hline Anaemia prevalence & $68.7(389)$ \\
\hline Microcytosis & $48.9(277)$ \\
\hline Malnutrition & $15.2(86)$ \\
\hline
\end{tabular}

${ }^{a}$ Calculated for 407 participants.

were, respectively, $62.4 \%, 27.7 \%, 48.9 \%, 58.4 \%, 27.7 \%$, and $15.2 \%$.

3.2. Malaria Parasite, Nutritional Status, and Dietary Habits. Significantly higher prevalence of malaria parasite was observed in children aged $6-10$ years $(35.5 \%, P=0.003)$, those from low altitude $(32.8 \%, P=0.001)$, and those who presented themselves at the hospital $(33.5 \%, P<0.001)$ when compared with their respective contemporaries. The prevalence of malaria parasite and malnutrition was comparable with sex $(P=0.715$ and $P=0.421$, respectively). However, significantly higher occurrence of malnutrition was observed in children aged $1-5$ years $(21.0 \% ; P<0.001)$, those living at low altitude $(18.1 \% ; P=0.015)$, those whose parents were fishermen $(23.3 \%, P=0.025)$, and those of other ethnicity (26.5\%; $P=0.029)$ as shown in Table 2 .

On dietary habits, more children $\leq 5$ years reported not washing hands before meals (19.9\%) and consuming fewer plantain meals a week than their older peers. This difference was statistically significant ( $P=0.001$ and $P=0.038$, respectively). The weekly plantain consumption was significantly linked to parents' education level $(P=0.049)$ with more children whose parents had no formal education consuming plantain $\geq 3$ times weekly $(55.8 \%)$ when compared with their counterparts as shown in Figure 1.

3.3. Anaemia, Microcytic Anaemia, and Malarial Anaemia Prevalence. Overall, the prevalence of anaemia (68.7\%) was highest in children living in low altitude (77.3\%), examined in the community (81.2\%), and living in homes with 6-10 occupants (71.4\%) and whose parents were 25-35 years old (80.2\%), had no formal education $(75.8 \%)$, or were fishermen $(86.4 \%)$ as well as those of forest ethnicity (100.0\%) when compared with their compeers. The differences were statistically significant. In relation to clinical factors, the only significantly higher prevalence of anaemia $(P=0.011)$ was observed in those with inflammation (79.1\%) as shown in Table 3.

The prevalence of microcytic anaemia was $36.9 \%$ accounting for $53.7 \%$ of all the anaemic cases. Significantly, it was highest in children $\leq 5$ years old $(43.3 \%)$, those living in low altitude $(49.1 \%)$, those examined within the community (42.8\%), those whose parents were $26-35$ years old $(39.7 \%)$ and were fishermen (56.3\%), those of forest ethnicity $(60.0 \%)$, those malaria parasite positive $(49.7 \%)$, and those who had moderate parasitaemia $(62.8 \%)$ when compared with their respective contemporaries. While malarial anaemia occurred in $19.6 \%$ of the study population, children aged $6-10$ years (24.1\%), those living at low altitude (23.9\%), those whose parents were $26-35$ years old (21.6\%), and those who were feverish $(28.7 \%)$ and were malaria parasite positive $(68.8 \%)$ had significantly higher prevalence than their respective peers (Table 3 ).

3.4. Effect of Nutritional Status and Dietary Habits on the Different Types of Anaemia. The prevalence of anaemia was significantly higher $(P=0.010, P=0.047$, and $P=0.009)$ in children who were stunted $(83.1 \%)$, were underweight (89.5\%), and consumed plantains 3-4 times a week (85.3\%), respectively, when compared with their equivalents. On the other hand, microcytic anaemia prevalence was significantly higher $(P=0.048)$ in normal $(43.7 \%)$ than wasted children, while only those who consumed plantains 3-4 times a week had a significantly higher $(P=0.025)$ prevalence of malarial anaemia $(20.6 \%)$ as shown in Table 4.

3.5. Risk Factors for Anaemia, Microcytic Anaemia, and Malarial Anaemia. As revealed in Table 5, the logistic regression analysis with anaemia as dependent variable showed that children living in lowland $(P=0.017)$, those 
TABLe 2: Prevalence of malaria parasite and malnutrition by sociodemographic factors.

\begin{tabular}{|c|c|c|c|c|c|c|}
\hline Parameter & Category & $N$ & $\begin{array}{c}\text { Malaria parasite prevalence } \% \\
(n)\end{array}$ & $\begin{array}{c}\chi^{2} \\
P \text { value }\end{array}$ & $\begin{array}{l}\text { Malnutrition } \\
\text { prevalence }\end{array}$ & $\begin{array}{c}\chi^{2}, \\
P \text { value } \\
\end{array}$ \\
\hline \multirow{2}{*}{ Gender } & Male & 267 & $28.5(76)$ & \multirow{2}{*}{$\begin{array}{c}0.133 \\
0.715\end{array}$} & $16.5(44)$ & \multirow{2}{*}{$\begin{array}{c}0.648 \\
0.421\end{array}$} \\
\hline & Female & 299 & $27.1(81)$ & & $14.0(42)$ & \\
\hline \multirow{3}{*}{ Age } & $1-5$ & 291 & $28.9(84)$ & \multirow{3}{*}{$\begin{array}{c}11.855 \\
0.003\end{array}$} & $21.0(61)$ & \multirow{3}{*}{$\begin{array}{l}17.110 \\
<0.001\end{array}$} \\
\hline & $6-10$ & 141 & $35.5(50)$ & & $6.4(9)$ & \\
\hline & $11-15$ & 134 & $17.2(23)$ & & $11.9(16)$ & \\
\hline \multirow{2}{*}{ Altitude } & Lowland & 348 & $32.8(114)$ & \multirow{2}{*}{$\begin{array}{c}11.360 \\
0.001\end{array}$} & $18.1(63)$ & 5.934 \\
\hline & Highland & 218 & $19.7(43)$ & & $10.6(23)$ & 0.015 \\
\hline \multirow{2}{*}{$\begin{array}{l}\text { Point of } \\
\text { presentation }\end{array}$} & Community & 208 & $17.8(37)$ & \multirow{2}{*}{$\begin{array}{l}16.243 \\
<0.001\end{array}$} & $17.8(37)$ & 1.717 \\
\hline & Hospital & 358 & $33.5(120)$ & & $13.7(49)$ & 0.190 \\
\hline \multirow{4}{*}{ Parents' age ${ }^{c}$} & $\leq 25$ & 16 & $6.2(1)$ & \multirow{4}{*}{$\begin{array}{l}3.940 \\
0.268\end{array}$} & $25.0(4)$ & \multirow{4}{*}{$\begin{array}{l}5.076 \\
0.166\end{array}$} \\
\hline & $26-35$ & 116 & $22.4(26)$ & & $17.2(20)$ & \\
\hline & $36-50$ & 180 & $20.6(37)$ & & $13.9(25)$ & \\
\hline & $>50$ & 95 & $14.4(14)$ & & $8.4(8)$ & \\
\hline \multirow{4}{*}{ Education } & Primary & 132 & $19.7(26)$ & \multirow{4}{*}{$\begin{array}{l}7.288 \\
0.063\end{array}$} & $17.4(23)$ & \multirow{4}{*}{$\begin{array}{l}2.516 \\
0.472\end{array}$} \\
\hline & Secondary & 230 & $28.3(65)$ & & $13.9(32)$ & \\
\hline & Tertiary & 179 & $31.3(56)$ & & $14.0(25)$ & \\
\hline & No formal & 25 & $40.0(10)$ & & $24.0(6)$ & \\
\hline \multirow{3}{*}{ Family size } & $1-5$ & 286 & $32.5(93)$ & \multirow{3}{*}{$\begin{array}{l}6.605 \\
0.037\end{array}$} & $13.6(39)$ & \multirow{3}{*}{$\begin{array}{l}2.629 \\
0.269\end{array}$} \\
\hline & $6-10$ & 248 & $23.0(57)$ & & $17.7(44)$ & \\
\hline & $>10$ & 32 & $21.9(7)$ & & $9.4(3)$ & \\
\hline \multirow{7}{*}{ Occupation } & Farming & 123 & $29.3(36)$ & \multirow{7}{*}{$\begin{array}{c}11.239 \\
0.081\end{array}$} & $10.6(13)$ & \multirow{7}{*}{$\begin{array}{c}14.495 \\
0.025\end{array}$} \\
\hline & Civil servant & 77 & 24.7 (19) & & $10.4(8)$ & \\
\hline & Petty trading & 63 & $33.3(21)$ & & $9.5(6)$ & \\
\hline & Private worker & 92 & $20.7(19)$ & & $16.3(15)$ & \\
\hline & Fishing & 103 & $28.2(29)$ & & $23.3(24)$ & \\
\hline & Jobless & 69 & $39.1(27)$ & & $23.2(16)$ & \\
\hline & Retired & 39 & $15.4(6)$ & & $10.3(4)$ & \\
\hline \multirow{5}{*}{ Ethnicity } & Coastal & 212 & $19.3(41)$ & \multirow{5}{*}{$\begin{array}{l}2.861 \\
0.581\end{array}$} & $10.8(23)$ & \multirow{5}{*}{$\begin{array}{c}10.816 \\
0.029\end{array}$} \\
\hline & Grassland & 112 & $17.0(19)$ & & $12.5(14)$ & \\
\hline & Forest & 5 & $20.0(1)$ & & $20.0(1)$ & \\
\hline & Sahel & 7 & $0.0(0)$ & & $14.3(1)$ & \\
\hline & $\begin{array}{l}\text { Other ethnic } \\
\text { groups }\end{array}$ & 68 & $23.5(16)$ & & $26.5(18)$ & \\
\hline
\end{tabular}

${ }^{c}$ Calculated for 407 participants. $P$ values in bold are statistically significant.

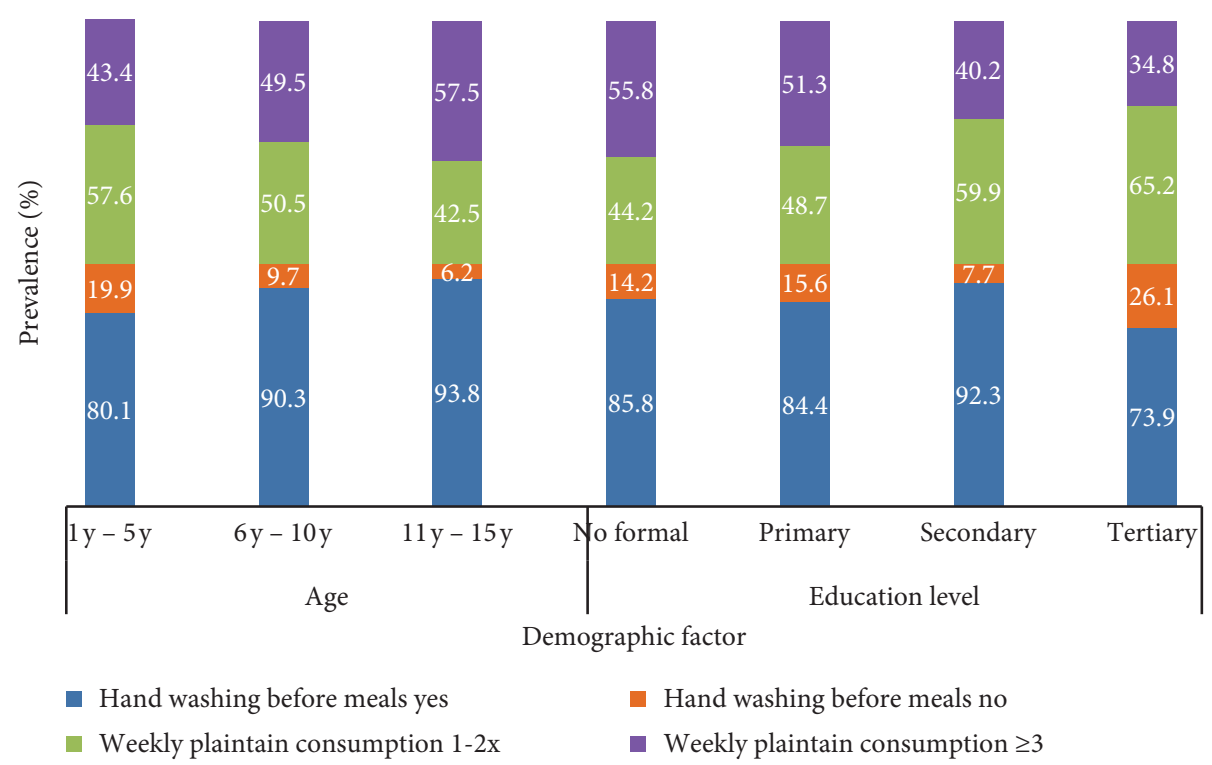

Figure 1: Effect of age and parents' education level on dietary habits. 
TABle 3: Prevalence of anaemia, microcytic anaemia, and malarial anaemia with respect to sociodemographic and clinical factors.

\begin{tabular}{|c|c|c|c|c|c|c|c|c|}
\hline \multirow{2}{*}{ Parameter } & \multirow{2}{*}{ Category } & \multirow{2}{*}{$N$} & \multicolumn{6}{|c|}{ Prevalence $[\%(n)]$ of } \\
\hline & & & Anaemia & $P$ value & Microcytic anaemia & $P$ value & Malarial anaemia & $P$ value \\
\hline \multicolumn{9}{|c|}{ Sociodemographic factors } \\
\hline \multirow{3}{*}{ Gender } & Male & 267 & $70.0(187)$ & & $39.7(106)$ & \multirow{3}{*}{0.196} & $20.6(55)$ & \multirow{3}{*}{0.57 .6} \\
\hline & Female & 299 & $67.6(202)$ & 0.525 & $34.4(103)$ & & $18.7(56)$ & \\
\hline & $1-5$ & 291 & $69.1(201)$ & & $43.3(126)$ & & $21.6(63)$ & \\
\hline \multirow[t]{2}{*}{ Age } & $6-10$ & 141 & $70.2(99)$ & 0.781 & $38.3(54)$ & $<0.001$ & $25.5(35)$ & \multirow{3}{*}{0.001} \\
\hline & $11-15$ & 134 & $66.4(89)$ & & $21.6(29)$ & & $9.0(12)$ & \\
\hline \multirow{2}{*}{ Altitude } & Lowland & 348 & $77.3(269)$ & & $49.1(179)$ & & $24.4(85)$ & \\
\hline & Highland & 218 & $55.0(120)$ & $<0.001$ & $17.4(38)$ & $<0.001$ & $11.9(26)$ & \multirow[t]{2}{*}{$<0.001$} \\
\hline \multirow{2}{*}{ Point of presentation } & Community & 208 & 81.2 (169) & & $42.8(89)$ & & $15.9(33)$ & \\
\hline & Hospital & 358 & $61.5(220)$ & $<0.001$ & $33.5(120)$ & 0.028 & $21.8(78)$ & \multirow[t]{2}{*}{0.089} \\
\hline \multirow{5}{*}{ Parents' age ${ }^{c}$} & $\leq 25$ & 16 & $68.8(11)$ & & $12.5(2)$ & & $6.2(1)$ & \\
\hline & $26-35$ & 116 & $80.2(93)$ & 0.006 & 39.7 (46) & 0.004 & $21.6(25)$ & \multirow[t]{4}{*}{0.046} \\
\hline & $36-50$ & 180 & 70.6 (127) & & $31.1(56)$ & & $12.8(23)$ & \\
\hline & $>50$ & 95 & $57.9(55)$ & & $18.9(18)$ & & $9.5(9)$ & \\
\hline & $1-5$ & 286 & 69.6 (199) & & 40.9 (117) & & $21.7(62)$ & \\
\hline \multirow[t]{3}{*}{ Family size } & $6-10$ & 248 & 71.4 (177) & 0.002 & $33.9(84)$ & 0.086 & $17.3(43)$ & \multirow[t]{3}{*}{0.449} \\
\hline & $>10$ & 32 & $40.6(13)$ & & $25.0(8)$ & & $18.8(6)$ & \\
\hline & Primary & 132 & $75.8(100)$ & & $40.2(53)$ & & $13.6(18)$ & \\
\hline \multirow{3}{*}{ Education } & Secondary & 230 & 73.5 (169) & 0.001 & $39.1(90)$ & 0.306 & $20.9(48)$ & \multirow[t]{3}{*}{0.057} \\
\hline & Tertiary & 179 & $57.0(102)$ & & $31.3(56)$ & & $20.1(36)$ & \\
\hline & No formal & 25 & $72.0(18)$ & & $40.0(10)$ & & $36.0(9)$ & \\
\hline \multirow{7}{*}{ Occupation } & Farming & 123 & $71.5(88)$ & & $39.0(48)$ & & $18.7(23)$ & \\
\hline & Civil servant & 77 & $57.1(44)$ & & $18.2(14)$ & & $14.3(11)$ & \\
\hline & Petty trading & 63 & $52.4(33)$ & & $25.4(16)$ & & $19.0(12)$ & \\
\hline & Private worker & 92 & $76.1(70)$ & & $35.9(33)$ & & $16.3(15)$ & \\
\hline & Fishing & 103 & $86.4(89)$ & $<0.001$ & $56.3(58)$ & $<0.001$ & $24.3(25)$ & 0.094 \\
\hline & Jobless & 69 & $63.8(44)$ & & $46.4(32)$ & & $30.4(21)$ & \\
\hline & Retired & 39 & $53.8(21)$ & & $20.5(8)$ & & $10.3(4)$ & \\
\hline & Coastal & 212 & $68.9(146)$ & & $27.8(59)$ & & $12.7(27)$ & \\
\hline & Grassland & 112 & $61.6(69)$ & & $23.2(26)$ & & $15.2(17)$ & \\
\hline Ethnicity & Forest & 5 & $100.0(5)$ & 0.002 & $60.0(3)$ & 0.002 & $20.0(1)$ & 0.546 \\
\hline & Sahel & 7 & $71.4(5)$ & & $14.3(1)$ & & $0.0(0)$ & \\
\hline & Other ethnic groups & 68 & $88.2(60)$ & & $48.5(33)$ & & $19.1(13)$ & \\
\hline & & & Clinical fac & tors & & & & \\
\hline & No & 409 & $69.2(283)$ & & $35.2(144)$ & & $15.4(63)$ & \\
\hline Fever & Yes & 157 & $67.5(106)$ & 0.700 & $41.4(65)$ & 0.172 & $30.6(48)$ & $<0.001$ \\
\hline Malaria parasite status & Positive & 157 & $70.7(111)$ & & $49.7(78)$ & & $70.7(111)$ & \\
\hline Mrararia paraste status & Negative & 409 & $60.0(278)$ & 0.531 & $32.0(131)$ & $<0.001$ & $0.0(0)$ & $<0.001$ \\
\hline & Low & 92 & $70.7(65)$ & & $50.0(46)$ & & $70.7(65)$ & \\
\hline Parasite load & Moderate & 43 & $74.4(32)$ & 0.665 & $62.8(27)$ & 0.009 & $74.4(32)$ & 0.665 \\
\hline & High & 22 & $63.6(14)$ & & $22.7(5)$ & & $63.3(14)$ & \\
\hline & No & 480 & $11.3(20)$ & 0.082 & $14.6(52)$ & 0.586 & $14.7(67)$ & 0.529 \\
\hline Mrainutrition & Yes & 86 & $17.0(66)$ & & $16.3(34)$ & & 17.1 (19) & \\
\hline Inflammation & No & 150 & $67.3(101)$ & 0.011 & $44.7(67)$ & 0.436 & $22.7(34)$ & 0.902 \\
\hline & Yes & 211 & $79.1(167)$ & & $48.8(103)$ & & $23.2(49)$ & \\
\hline
\end{tabular}

${ }^{c}$ Calculated for 407 participants: $P$ value obtained by $\chi^{2}, P$ values in bold are statistically significant.

whose parents were farmers, worked privately, or were fishermen $(P=0.042 ; P=0.013 ; P<0.001$, respectively $)$, and those who consumed meat 3-4 times weekly $(P=0.026)$ and plantains $(P=0.034)$ were significantly at risk of developing anaemia. They were, respectively, 4, 2.2, 2.7, 5.5, 2.5 and 2.5 times more likely to be anaemic than their counterparts. Significantly, children aged $1-5$ years $(P=0.007)$, those of forest ethnicity $(P=0.007)$, those whose parents are farmers $(P=0.038)$ or jobless $(P=0.009)$, and those who had moderate parasitaemia $(P=0.048)$ were $2.4,15.7,2.5$, 3.4 , and 2.6 times, respectively, more likely to have microcytic anaemia. With respect to malarial anaemia, the identified risk factors were the child's age $(P=0.008)$, parent's age $(P=0.049)$ and occupation $(P=0.023)$, and child's weekly consumption of plantain $(P=0.024)$. Children aged 6-10 years, whose parents were $26-35$ years old or 
TAвLe 4: Prevalence of anaemia, microcytic anaemia, and malarial anaemia with respect to nutritional status and dietary habits.

\begin{tabular}{|c|c|c|c|c|c|c|c|c|}
\hline \multirow{2}{*}{ Parameter } & \multirow{2}{*}{ Status } & \multirow{2}{*}{$N$} & \multicolumn{6}{|c|}{ Prevalence $[\%(n)]$ of } \\
\hline & & & Anaemia & $P$ value & Microcytic anaemia & $P$ value & Malarial anaemia & $P$ value \\
\hline \multirow{2}{*}{ Stunting } & No & 494 & $66.6(329)$ & & $35.0(173)$ & & $18.8(93)$ & \\
\hline & Yes & 59 & $83.1(49)$ & 0.010 & $47.5(28)$ & 0.060 & $23.7(14)$ & 0.368 \\
\hline \multirow{2}{*}{ Underweight } & No & 405 & $67.9(275)$ & & $40.7(165)$ & & $22.7(92)$ & \\
\hline & Yes & 19 & $89.5(17)$ & 0.047 & $47.4(9)$ & 0.566 & $21.1(4)$ & 0.866 \\
\hline \multirow{2}{*}{ Wasting } & No & 249 & $67.5(168)$ & & $43.7(107)$ & & $21.7(54)$ & \\
\hline & Yes & 23 & $60.9(14)$ & 0.520 & $21.7(5)$ & 0.048 & $13.0(3)$ & 0.330 \\
\hline \multirow{3}{*}{ Hand washing before meal } & Yes & 352 & $68.8(242)$ & & $28.4(100)$ & & $13.6(48)$ & \\
\hline & No/seldom & 55 & $80.0(44)$ & 0.090 & $40.0(22)$ & 0.081 & $18.2(10)$ & 0.370 \\
\hline & 1 & 41 & $80.5(33)$ & & $39.0(16)$ & & $19.5(8)$ & \\
\hline \multirow[t]{3}{*}{ Daily meal } & 2 & 146 & $70.5(103)$ & & $30.8(45)$ & & $13.7(20)$ & \\
\hline & 3 & 220 & $68.2(150)$ & 0.285 & $27.7(61)$ & 0.336 & $13.6(30)$ & 0.597 \\
\hline & $1-2$ & 173 & $74.6(129)$ & & $28.9(50)$ & & $15.6(27)$ & \\
\hline \multirow[t]{3}{*}{ Weekly fruits consumption } & $3-4$ & 57 & $57.9(33)$ & & $31.6(18)$ & & $14.0(8)$ & \\
\hline & $>4$ & 177 & $70.1(124)$ & 0.058 & $30.5(54)$ & 0.910 & $13.0(23)$ & 0.782 \\
\hline & $1-2$ & 178 & $69.7(124)$ & & $29.2(52)$ & & $15.2(27)$ & \\
\hline \multirow[t]{3}{*}{ Weekly meat consumption } & $3-4$ & 59 & $83.1(49)$ & & $37.3(22)$ & & $20.3(12)$ & \\
\hline & $>4$ & 170 & $66.5(113)$ & 0.054 & $28.2(48)$ & 0.407 & $11.2(19)$ & 0.199 \\
\hline & $1-2$ & 210 & $65.7(138)$ & & $28.6(60)$ & & $16.2(34)$ & \\
\hline \multirow[t]{2}{*}{ Weekly plantain consumption } & $3-4$ & 68 & $85.3(58)$ & & $25.0(17)$ & & $20.6(14)$ & \\
\hline & $>4$ & 129 & $69.8(90)$ & 0.009 & 34.9 (45) & 0.289 & 7.8 (10) & 0.025 \\
\hline
\end{tabular}

$P$ values in bold are statistically significant.

jobless, and who consumed plantains 3-4 times a week were $3.3,2.6,3.8$, and 2.9 times, respectively, more likely to have malarial anaemia than their counterparts.

\section{Discussion}

This study investigates the prevalence and risk factors for microcytic and malarial anaemia in children 1-15 years in urban settings of the Mount Cameroon area. The overall malaria prevalence of $27.7 \%$ is like that reported by Teh et al. [33] with the highest burden (35.5\%) observed in children in the 6-10 years age group and those living in low altitude. The shift in malaria burden from the under-fives to the 6-10 years observed in this study may be due to effective bed net use in the former corroborating findings elsewhere [34].

More than half of the study participants reported washing hands before meal and consuming plantains more than twice weekly. Hand washing increased with age because younger children must be reminded frequently to wash their hands but will form the habit as they grow older. This finding is corroborated by studies elsewhere [35]. In addition, findings from the study revealed that plantain consumption increased with child's age but decreased with parent's level of education. Younger children may prefer the ripe version of the plantain or paste, which is easier for them to chew/ swallow but contains more carbohydrates, to the harder unripe plantain [36] consumed easily by older children which has more of iron and vitamin C. On the other hand, increase in the level of schooling increases knowledge on better feeding practices and dietary diversity [37, 38]; also income may account for the decrease in plantain consumption with rise in level of education as participants can probably afford to replace plantains with other iron-rich foods. That notwithstanding, unripe plantains are rich in iron and vitamin $\mathrm{C}$ which are two micronutrients that optimize absorption and can aid in avoiding the occurrence of anaemia [39].

The overall anaemia prevalence of $68.7 \%$ reveals that anaemia is a serious public health problem in children in this region as elsewhere in the country. This is higher than both the national anaemia prevalence of $60 \%$ [9] and that observed by Teh et al. [34] in the same area but lower than the $77.2 \%$ reported in Tanzania [111]. The higher prevalence of anaemia among the 6-10 years old compared with their counterparts is not a surprise as a similar higher prevalence of malaria was observed in the same group. Malaria parasite infection has been reported severally as a risk factor of anaemia $[21,22,34]$. In addition, observations from studies in the Mount Cameroon area have revealed that the burden of malaria has shifted from the $\leq 5$ years old to older age groups due to the increased preventive measures in the former group [34, 40, 41]. This age-related change in burden of malaria may have led to the shift in anaemia prevalence from the under-fives to older groups.

That anaemia was more prevalent at lower (Limbe municipality) than higher altitude (Buea municipality) in this study shows the association between malaria, anaemia, and altitude which had long been established in previous studies [11,33, 42]. Children living at lower altitude were 4 times at odds of being anaemic. Higher temperatures at low altitudes favour breeding of the malaria vector and transmission of malaria parasites [43-45] which in turn leads to increased anaemia prevalence as malaria has been shown to be among the major contributors to the occurrence of anaemia in this setting [34].

Contrary to findings by Sakwe et al. [45] who reported lower anaemia prevalence in a community-based study than 
TABLE 5: Multinomial regression analysis examining sociodemographic, clinical, hygiene, and dietary factors associated with anaemia, microcytic anaemia, and malarial anaemia.

\begin{tabular}{|c|c|c|c|c|c|c|c|}
\hline \multirow{2}{*}{ Parameter } & \multirow{2}{*}{ Category } & \multicolumn{2}{|c|}{ Anaemia } & \multicolumn{2}{|c|}{ Microcytic anaemia } & \multicolumn{2}{|c|}{ Malarial anaemia } \\
\hline & & $\operatorname{AOR}(95 \% \mathrm{CI})$ & $P$ value & AOR $(95 \% \mathrm{CI})$ & $P$ value & AOR $(95 \% \mathrm{CI})$ & $P$ value \\
\hline \multicolumn{8}{|c|}{ Sociodemographic factors } \\
\hline \multirow{3}{*}{ Gender } & Male & $1.0(0.6-1.6)$ & 0.904 & $1.2(0.7-2.0)$ & 0.417 & $0.7(0.4-1.4)$ & 0.355 \\
\hline & Female & Reference & & Reference & & Reference & \\
\hline & $1-5$ & $0.9(0.5-1.6)$ & 0.685 & $2.4(1.3-4.6)$ & 0.007 & $1.9(0.8-4.5$ & 0.163 \\
\hline \multirow[t]{2}{*}{ Age } & $6-10$ & $1.1(0.5-2.1)$ & 0.852 & $2.0(1.0-3.9)$ & 0.060 & $3.3(1.4-8.1)$ & 0.008 \\
\hline & $11-15$ & Reference & & Reference & & Ref & \\
\hline \multirow{2}{*}{ Altitude } & Lowland & $4.0(1.3-12.4)$ & 0.017 & $1.7(0.5-6.1)$ & 0.401 & $3.3(0.8-13.6)$ & 0.096 \\
\hline & Highland & Reference & & Reference & - & Reference & - \\
\hline \multirow{3}{*}{ Presentation } & Hospital & $1.5(0.5-4.4)$ & 0.515 & $0.4(0.1-1.4)$ & 0.133 & $2.8(0.7-11.5)$ & 0.147 \\
\hline & Community & Reference & - & Reference & - & Reference & - \\
\hline & $>10$ & $0.2(0.1-0.7)$ & 0.017 & $1.2(0.4-4.7)$ & 0.748 & $1.0(0.3-4.1)$ & 0.979 \\
\hline \multirow[t]{3}{*}{ Family size } & $6-10$ & $1.1(0.6-1.8)$ & 0.828 & $1.1(0.6-1.8)$ & 0.833 & $0.8(0.4-1.5)$ & 0.446 \\
\hline & $1-5$ & Reference & & Reference & - & Reference & - \\
\hline & $\leq 25$ & $1.0(0.3-3.7)$ & 0.991 & $0.5(0.1-2.4)$ & 0.349 & $0.8(0.1-7.1)$ & 0.826 \\
\hline \multirow{4}{*}{ Parents' age } & $26-35$ & $1.8(0.9-4.0)$ & 0.117 & $1.7(0.8-3.7)$ & 0.163 & $2.6(1.0-6.6)$ & 0.049 \\
\hline & $36-50$ & $1.2(0.6-2.2)$ & 0.645 & $1.8(0.9-3.8)$ & 0.105 & $1.4(0.6-3.4)$ & 0.481 \\
\hline & $>50$ & Reference & - & Reference & - & Reference & - \\
\hline & No formal & $1.9(0.6-5.7)$ & 0.261 & $0.6(0.2-1.8)$ & 0.388 & $0.2(0.1-0.6)$ & 0.005 \\
\hline \multirow{3}{*}{ Education level } & Primary & $1.5(0.5-4.4)$ & 0.506 & $0.5(0.2-1.5)$ & 0.215 & $0.2(0.1-0.6)$ & 0.003 \\
\hline & Secondary & $1.1(0.3-3.4)$ & 0.902 & $0.5(0.2-1.8)$ & 0.303 & $0.3(0.1-0.9)$ & 0.030 \\
\hline & Tertiary & Reference & - & Reference & - & Reference & - \\
\hline \multirow[t]{5}{*}{ Ethnicity } & Coastal & $1.4(0.4-5.5)$ & 0.645 & $1.4(0.4-4.3)$ & 0.599 & $0.6(0.3-1.3)$ & 0.194 \\
\hline & Grassland & $2.2(0.6-8.5)$ & 0.265 & $0.8(0.3-2.5)$ & 0.701 & $0.8(0.3-1.7)$ & 0.493 \\
\hline & Forest & - & - & $15.7(1.6-155.4)$ & 0.019 & $1.1(0.1-10.3)$ & 0.961 \\
\hline & Sahel & $4.3(0.4-41.7)$ & 0.210 & $0.1(0.0-1.5)$ & 0.098 & & - \\
\hline & Others & Reference & - & Reference & & Reference & - \\
\hline \multirow[t]{7}{*}{ Occupation } & Farming & $2.2(1.0-4.5)$ & 0.042 & $2.5(1.1-5.9)$ & 0.038 & $2.0(0.7-6.2)$ & 0.225 \\
\hline & Civil servant & $1.1(0.5-2.5)$ & 0.735 & $0.9(0.3-2.3)$ & 0.762 & $1.5(0.4-4.9)$ & 0.543 \\
\hline & Petty trading & $0.9(0.4-2.1)$ & 0.885 & $1.3(0.5-3.5)$ & 0.573 & $2.1(0.6-56.9)$ & 0.242 \\
\hline & Private worker & $2.7(1.2-6.0)$ & 0.013 & $2.2(0.9-5.3)$ & 0.087 & $1.7(0.5-5.5)$ & 0.373 \\
\hline & Fishing & $5.5(2.3-12.7)$ & $<0.001$ & $0.3(0.0-1.5)$ & $<0.001$ & $2.8(0.9-8.7)$ & 0.073 \\
\hline & Jobless & $1.5(0.7-3.4)$ & 0.313 & $3.4(1.4-8.3)$ & 0.009 & $3.8(1.2-12.1)$ & 0.023 \\
\hline & Retired & Reference & - & Reference & & Reference & - \\
\hline \multirow{3}{*}{ Malnutrition } & & Clinico & factors & & & & \\
\hline & Yes & $1.2(0.1-31.4)$ & 0.903 & $0.2(0.0-2.3)$ & 0.196 & $1.2(0.0-31.4)$ & 0.903 \\
\hline & No & Reference & & Reference & & Reference & \\
\hline Stunting & Yes & $0.4(0.0-12.5)$ & 0.621 & $7.9(0.7-84.2)$ & 0.088 & $0.4(0.0-12.5)$ & 0.621 \\
\hline & No & Reference & & Reference & & Reference & - \\
\hline Underweight & Yes & $0.7(0.0-12.2)$ & 0.831 & $2.3(0.6-9.5)$ & 0.245 & $0.7(0.0-12.2)$ & 0.831 \\
\hline Underweight & No & Reference & & Reference & - & Reference & - \\
\hline & Yes & - & - & $1.4(0.1-12.2)$ & 0.912 & - & - \\
\hline Wasting & No & Reference & & Reference & - & Reference & - \\
\hline & High & $0.3(0.1-1.8)$ & 0.183 & $0.1(0.0-1.2)$ & 0.071 & $0.1(0.0-1.9)$ & 0.129 \\
\hline Parasitaemia & Moderate & $0.2(0.0-1.3)$ & 0.086 & $2.6(1.0-6.6)$ & 0.048 & $0.9(0.2-3.9)$ & 0.932 \\
\hline & Low & Ref & & Reference & & Ref & \\
\hline & Yes & $1.1(0.3-4.3)$ & 0.937 & $2.0(0.9-4.4)$ & 0.084 & $1.1(0.3-4.3)$ & 0.937 \\
\hline Inflammation & No & Ref & & Ref & & Ref & \\
\hline & & Dietar & factors & & & & \\
\hline Weekly fruit consumption & $1-2$ & $1.4(0.8-2.4)$ & 0.240 & $1.0(0.6-1.7)$ & 0.995 & $0.9(0.5-1.9)$ & 0.861 \\
\hline & $3-4$ & $0.4(0.2-0.8)$ & 0.015 & $1.1(0.6-2.2)$ & 0.783 & $0.7(0.3-1.8)$ & 0.471 \\
\hline & $>4$ & Reference & & Reference & - & Reference & - \\
\hline Weakly meat consumption & $1-2$ & $1.2(0.7-2.1)$ & 0.430 & $1.1(0.7-1.9)$ & 0.661 & $1.3(0.7-2.6)$ & 0.436 \\
\hline & $3-4$ & $2.5(1.1-5.6)$ & 0.026 & $1.8(0.9-3.6)$ & 0.077 & $1.6(0.7-3.8)$ & 0.346 \\
\hline & $>4$ & Reference & & Reference & - & Reference & - \\
\hline Weekly Plantain consumption & $1-2$ & $0.7(0.4-1.2)$ & 0.174 & $0.7(0.2-1.2)$ & 0.158 & $2.2(1.0-4.8)$ & 0.052 \\
\hline & $3-4$ & $2.5(1.1-5.6)$ & 0.034 & $0.5(0.2-1.0)$ & 0.057 & $2.9(1.2-7.3)$ & 0.024 \\
\hline & $>4$ & Reference & - & Reference & - & Reference & \\
\hline
\end{tabular}

$P$ values in bold are statistically significant. 
in sick children seeking treatment in the hospital, our findings revealed that anaemia was more present in children within the community than at presentation to hospital. This may be due in part to the occurrence of submicroscopic Plasmodium infection that was not determined and the prevalence of malaria parasite observed in these apparently healthy children in the community. It is established that malaria causes haemolysis of both parasitized and healthy red cells leading to anaemia [13]. Also, in malaria parasite intense transmission regions, apparently healthy children may have subclinical infection even in the absence of overt disease. Effect of these subclinical infections are usually determined by measuring the concentration of acute phase proteins such as CRP involved in the inflammatory response. Inflammation being primarily protective [46] also causes the retention of iron by the reticuloendothelial macrophages leading to lower iron concentration in circulation and thus anaemia [47].

Parents' age, education level, occupation, and ethnicity as well as family size are sociodemographic factors that are intrinsically linked and significantly associated with childhood anaemia as reported in previous studies [38, 48, 49]. Parents who were $\leq 35$ years old and those with no formal education had more anaemic children than the other parents. Being $\leq 25$ years old may suggest that as first-time parents they are less prepared for parenthood and meeting the nutritional needs of the child. This may also be compounded by lack of knowledge on childcare and anaemia $[50,51]$. Also, because a parent has little or no formal education, their chances of getting skilled jobs are also very reduced, leading to low wages and limited access to healthy food sources [52]. The odds of having anaemia was lesser in children in homes with $>10$ occupants. Contrary to studies where people living in or in proximity to forested areas have normal haemoglobin levels [53], findings from this study reveal that children with forest origin had the highest prevalence of anaemia. It may be that being away from their area of origin keeps them away from wild game and diverse forest foods which have been shown to improve iron intake and limit anaemia $[54,55]$.

Although not significant, observations from this study reveal that children who were feverish, were malaria parasite positive, and had moderate parasitaemia were more anaemic than their respective counterparts supporting findings from elsewhere [30, 56]. Fever is known as a characteristic symptom of malaria even though nonspecific where other infections are possible [57]. The fever may have been caused by immune responses to malaria or some other underlying disease.

Malnutrition affects all strata of the society but mostly children are more vulnerable because of high nutritional demands required for proper growth [58]. Anaemia was more common in malnourished children and was significantly higher in stunted (83.1\%) and underweight (89.5\%) children than their counterparts. This finding is in line with studies carried out in Bangladesh by Rahman et al. [59]. However, nutritional deficiencies may not always be directly linked to anaemia but could be the cause that weakens the general health of the children exposing them to other anaemia-causing diseases. This is supported by Khanam et al. [60].

Regarding microcytosis and microcytic anaemia, an overall $48.9 \%$ of the study population had microcytosis while microcytic anaemia prevalence was $36.7 \%$. This proportion ranks it as a moderate public health concern according to WHO classification on anaemia burden [32]. This proportion of $36.7 \%$ is comparable to that observed in Tanzania [11] but lower than the $49.0 \%$ observed by Mah et al. [40] in children seeking treatment in the Yaounde Gynaeco-Obstetric and Paedriatric Hospital. Microcytosis is defined as a lower than normal MCV for age. It is indicative of iron deficiency, and iron deficiency is said to be responsible for half of all anaemia cases [61]. However, since we did not measure ferritin levels and other markers for iron status, we may have underestimated the true depiction of iron deficiency in the population.

Males had higher microcytic anaemia prevalence (70\%) than females although this observation may not be a surprise since the males equally had higher prevalence of malaria and anaemia than females. Age-wise, we would have expected children 6-10 years old to have the highest prevalence of microcytic anaemia as with both malaria and anaemia but that was not the case. Instead, the $\leq 5$ years old had significantly higher prevalence of microcytic anaemia than their counterparts. Microcytosis and anaemia each have varied aetiologies, the result of which in combination may lead to microcytic anaemia. The observed microcytic anaemia in this case may have been due to anaemia resulting from nutritional deficiency and not malaria, as children in this age have high iron needs for growth. Furthermore, microcytosis may have been due to the body's protective mechanism to wade off infection with malaria parasite [62], or resulting from some other anaemia-causing factor such as the thalassaemia trait, a common haemoglobinopathy in malaria endemic African populations. It may also result from bacterial, viral, or helminth infections which were not evaluated, as it was out of the scope of this investigation and thus a probable limitation of the study.

A significant association between occupation, ethnicity, and microcytic anaemia is like that observed with anaemia. Parents who were jobless, fishermen, and farmers had children with significantly higher prevalence of microcytic anaemia than their counterparts of other occupation. It is understandable that being jobless predisposes one to lower income and therefore inadequate financial resources to provide nutritious meals to meet the daily nutrient requirement of the household. However, children whose parents/guardians were fishermen and farmers were expected not to have microcytic anaemia because they have access to iron-rich food sources. Nevertheless, observations from this study, congruent to previous studies in this area [21,34], show that many farmers do not consume the best of the foods they produce but rather sell them for money and consume the less presentable ones. Fish and vegetables are common sources of iron in the area which if consumed in the best state regularly alongside fruits will reduce the occurrence of anaemia. This reasserts reports from other studies $[63,64]$ that the low or nonconsumption of fruits, vegetables, and iron-rich animal sources increases risks for anaemia. 
The overall low prevalence of malarial anaemia in this population $(19.6 \%)$ reveals that malaria may not be a major contributor to the overall anaemia prevalence. This proportion is lower than the $27.7 \%$ previously observed in the same area [34]. Even though submicroscopic infection with Plasmodium falciparum infection has been associated with anaemia in other studies $[65,66]$ which was not investigated in the current study, the lack of association between malaria parasite density and anaemia likely highlights its limited contributions to the burden of anaemia in the study population. Of concern is the significant association of child's age, parent's age, education level, and occupation, with malarial anaemia in the regression analysis which may be directly linked to the influence of both malaria and anaemia. Febrility and malaria positivity influence on malarial anaemia prevalence cannot be overemphasized [21]. Nonetheless, fever, being a nonspecific symptom, may have also resulted from other infections which were not considered in this study.

While the findings of this study may be applicable in several areas in the region and elsewhere with similar environmental conditions and microclimates as well as cultural practices, there is a limitation in its applicability in areas nonendemic for malaria and having variable eating habits. However, the findings provide valuable information that could be used in the development of appropriate, context specific control measures against anaemia, microcytic anaemia, and malarial anaemia by public health authorities.

\section{Conclusions}

With anaemia prevalence still well above the cut-off value of $40 \%$, it is important to reevaluate childhood determinants that foster its presence and also revisit the implementation strategies that have so far been put in place. An anaemiamonitoring system, especially those aimed at reducing nutritional anaemia, will effectively help in curbing anaemia. While microcytic anaemia is closely moving to the red zone as a public health problem in urban areas of Mount Cameroon, malarial anaemia is of mild public health concern. The high microcytic anaemia prevalence in children aged $\leq 5$ years, those living at low altitude and within the community, shows that some insidious factors other than malaria are at work. While age, parent's/guardian's occupation and ethnicity, and moderate parasitaemia were risk factors for microcytic anaemia, malarial anaemia had as risk factors child's age, parent's age and occupation, and a $\leq 4$ times weekly consumption of plantains. Therefore, when planning interventions to reduce anaemia prevalence in the community, strategies that will elevate the socioeconomic status of the parents should be integrated to aid better and healthy food choices to reduce anaemia due to nutritional deficiencies.

\section{Data Availability}

All the datasets generated and analysed during the current study are presented in the paper.

\section{Conflicts of Interest}

The authors declare that they have no conflicts of interest.

\section{Acknowledgments}

The authors wish to thank the parents and children for their participation in this study. This study received no specific funding; however, laboratory investigation was partially supported by the special fund for research and modernisation given to the authors by the Government of Cameroon and the World Academy of Science (TWAS) grant awarded to one of the authors.

\section{References}

[1] S. S. Lim, T. Vos, A. D. Flaxman et al., "A comparative risk assessment of burden of disease and injury attributable to 67 risk factors and risk factor clusters in 21 regions, 1990-2010: a systematic analysis for the Global Burden of Disease Study 2010," The Lancet, vol. 380, no. 9859, pp. 2224-2260, 2012.

[2] N. J. Kassebaum, R. Jasrasaria, M. Naghavi et al., "A systematic analysis of global anemia burden from 1990 to 2010," Blood, vol. 123, no. 5, pp. 615-624, 2014.

[3] G. A. Stevens, M. M. Finucane, L. M. De-Regil et al., "Global, regional, and national trends in haemoglobin concentration and prevalence of total and severe anaemia in children and pregnant and non-pregnant women for 1995-2011: a systematic analysis of population-representative data," The Lancet Global Health, vol. 1, no. 1, pp. E16-E25, 2013.

[4] WHO, World Malaria Report, WHO Library Cataloguing-inPublication Data, Geneva, Switzerland, 2008.

[5] E. McLean, M. Cogswell, I. Egli, D. Wojdyla, and B. de Benoist, "Worldwide prevalence of anaemia, WHO vitamin and mineral nutrition information system, 1993-2005," Public Health Nutrition, vol. 12, no. 4, pp. 444-454, 2009.

[6] S. Horton and J. Ross, "The economics of iron deficiency," Food Policy, vol. 28, no. 1, pp. 51-75, 2003.

[7] L. Alcázar, "The economic impact of anaemia in Peru," in Group for the Analysis of Development and Action Against Hunger, GRADE, Lima, Peru, 2013.

[8] J. C. McCann and B. N. Ames, "An overview of evidence for a causal relation between iron deficiency during development and deficits in cognitive or behavioral function," The American Journal of Clinical Nutrition, vol. 85, no. 4, pp. 931-945, 2007.

[9] World Bank, "Prevalence of anaemia among children (\% of children under five)|data," 2020, https://data.worldbank.org/ indicator/SH.ANM.CHLD.ZS.

[10] Y. Balarajan, U. Ramakrishnan, E. Özaltin, A. H. Shankar, and S. Subramanian, "Anaemia in low-income and middle-income countries," The Lancet, vol. 378, no. 9809, pp. 2123-2135, 2011.

[11] R. H. Simbauranga, E. Kamugisha, A. Hokororo, B. R. Kidenya, and J. Makani, "Prevalence and factors associated with severe anaemia amongst under-five children hospitalized at Bugando Medical Centre, Mwanza, Tanzania," BMC Haematology, vol. 15, no. 13, 2015.

[12] M. H. Al-Mekhlafi, J. Surin, A. S. Atiya, W. A. Ariffin, A. K. M. Mahdy, and H. C. Abdullah, "Anaemia and iron deficiency anaemia among aboriginal schoolchildren in rural Peninsular Malaysia: an update on a continuing problem," Transactions of the Royal Society of Tropical Medicine and Hygiene, vol. 102, no. 10, pp. 1046-1052, 2008. 
[13] E. M. Al-Zabedi, F. A. Kaid, H. Sady, A. H. Al-Adhroey, A. A. Amran, and M. T. Al-Maktari, "Prevalence and risk factors of iron deficiency anemia among children in Yemen," American Journal of Health Research, vol. 2, no. 5, pp. 319326, 2014.

[14] T. N. William, "Red blood cell defects and malaria," Molecular Biochemistry and Parasitology, vol. 149, pp. 121-127, 2006.

[15] T. Ganz, "Molecular control of iron transport," Journal of the American Society of Nephrology, vol. 18, no. 2, pp. 394-400, 2007.

[16] C. J. Calis, K. S. Phiri, E. B. Faragher et al., "Severe anaemia in Malawian children," New England Journal of Medicine, vol. 358, no. 9, pp. 888-899, 2008.

[17] T. G. DeLoughery, "Microcytic anaemia," New England Journal of Medicine, vol. 371, pp. 324-331, 1992.

[18] J. Wallach, Interpretation of Diagnostic Tests, Little Brown and Company, Boston, MA, USA, 8th edition, 2006.

[19] R. Stoltzfus, "Update on issues related to iron deficiency and anaemia control," in Proceedings of the Report of the 2003 International Nutritional Anaemia Constative Group: Integrating Programs to Move Iron Deficiency Anaemia Control Forward Symposium, International Life Sciences Institute, Marrakech, Morocco, 2003.

[20] M. M. Sirdah, I. M. A. El-Agouza, and A. N. K. Abu Shahla, "Possible ameliorative effect of taurine in the treatment of iron-deficiency anaemia in female university students of Gaza, Palestine," European Journal of Haematology, vol. 69, no. 4, pp. 236-242, 2002.

[21] I. U. N Sumbele, S. O. Sama, H. K Kimbi, and G. S. Taiwe, "Malaria, moderate to severe anaemia, and malarial anaemia in children at presentation to hospital in the Mount Cameroon area: a cross-sectional study," Anemia, vol. 2016, Article ID 5725634, 12 pages, 2016.

[22] H. K. Kimbi, I. U. N. Sumbele, M. Nweboh et al., "Malaria and haematologic parameters of pupils at different altitudes along the slope of Mount Cameroon: a cross-sectional study," Malaria Journal, vol. 12, no. 1, 193 pages, 2013.

[23] S. G. Massoda Tonye, C. Kouambeng, R. Wounang, and P. Vounatsou, "Challenges of DHS and MIS to capture the entire pattern of malaria parasite risk and intervention effects in countries with different ecological zones: the case of Cameroon," Malaria Journal, vol. 17, no. 1, p. 156, 2018.

[24] N. M. C. National, Malaria Control Programme Annual Report SANTECameroon, MINSANTE, Yaounde, Cameroon, 2015.

[25] S. Wanji, T. Tanke, S. N. Atanga, C. Ajonina, T. Nicholas, and D. Fontenille, "Anopheles species of the mount Cameroon region: biting habits, feeding behaviour and entomological inoculation rates," Tropical Medicine and International Health, vol. 8, no. 7, pp. 643-649, 2003.

[26] N. Amvongo-Adjia, E. L. Wirsiy, J. M. Riveron et al., "Bionomics and vectorial role of anophelinesin wetlands along the volcanic chain of Cameroon," Parasites and Vectors, vol. 11, p. 471, 2018.

[27] F. J. Bryan, The Design and Analysis of Research Studies, Cambridge University Press, Cambridge, UK, 1992.

[28] WHO, "Malaria vector control and personal protection," vol. 936, WHO, Geneva, Switzerland, 2006, Technical Report Series.

[29] WHO, Anthro for Personal Computers, Software for Assessing Growth and Development of the World's Children, WHO, Geneva, Switzerland, 2010.
[30] M. Cheesbrough, District Laboratory Practice in Tropical Countries, Vol. 12, Cambridge University Press, Cambridge, UK, 2009.

[31] I. U. N. Sumbele, T. R. Ning, O. S. Bopda, and T. NkuoAkenji, "Variation in malariometric and red cell indices in children in the Mount Cameroon area following enhanced malaria control measures: evidence from a repeated crosssectional study," Malaria Journal, vol. 13, no. 334, 2014.

[32] WHO, Iron Deficiency Anaemia: Assessment, Prevention and control.A Guide for Programme Managers, World Health Organisation, Geneva, Switzerland, 2001.

[33] R. N. Teh, I. U. N. Sumbele, G. A. Nkeudem, D. N. Meduke, S. T. Ojong, and H. K. Kimbi, "Concurrence of CareStart ${ }^{\mathrm{TM}}$ Malaria HRP2 RDT with microscopy in population screening for Plasmodium falciparum infection in the Mount Cameroon area: predictors for RDT positivity," Tropical Medicine and Health, vol. 49, no. 17, 2019.

[34] R. N. Teh, I. U. N. Sumbele, D. N. Meduke, S. T. Ojong, and H. K. Kimbi, "Malaria parasitaemia, anaemia and malnutrition in children less than 15 years residing in different altitudes along the slope of Mount Cameroon: prevalence, intensity and risk factors," Malaria Journal, vol. 17, p. 336, 2018.

[35] Vanderbilt University, Teaching Your Child to Become Independent with Daily Routine, Vanderbilt University, Nashville, TN, USA, 2010.

[36] F. G. Honfo, A. Tenkouano, and O. Coulibaly, "Banana and plantain-based foods consumption by children and mothers in Cameroon and Southern Nigeria: a comparative study," African Journal of Food Science, vol. 5, no. 5, pp. 287-291, 2011.

[37] J. M. Morimoteo, M. R. D. O. Larorre, C. L. G. César et al., "Fatoresassociados à qualidade da dieta de adultosresidentesnaRegiaoametropolitana de São Paulo, Brasil," Cad SaùdePùblica, vol. 24, no. 1, pp. 169-78, 2002.

[38] D. B. Hipgrave, X. Fu, H. Zhou et al., "Poor complementary feeding practices and high anaemia prevalence among infants and young children in rural central and western China," European Journal of Clinical Nutrition, vol. 68, no. 8, pp. 916-924, 2014.

[39] M. Frey, "Plantain nutrition facts and health benefits," 2020, https://www.verywellfit.com/plantain-nutrition-factscalories-health-benefits.

[40] E. Mah, F. Nguefack, J. R. Nkeck, R. Mouto, A. H. Mbassi, and S. Nguefacketal, "Anaemia in children following an acute infectious illness: is systematic iron prescription justified?" Health, Science and Diseases, vol. 18, no. suppl. 3, 2017.

[41] C. Njua-Yafi, E. A. Achidi, J. K. Anchang-Kimbi et al., "Malaria, helminths, co-infection and anaemia in a cohort of children from Mutengene, south western Cameroon," Malaria Journal, vol. 15, no. 69, 2016.

[42] C. B. Ebai, H. K. Kimbi, I. U. N. Sumbele, J. E. Yunga, and L. G. Lehman, "Epidemiology of Plasmodium falciparum malaria in the Ikata-Likoko area of Mount Cameroon: a cross sectional study," International Journal of Tropical Disease and Health, vol. 16, no. 4, 2016.

[43] F. Kateera, C. M. Ingabire, E. Hakizimana et al., "Malaria, anaemia and under-nutrition: three frequently coexisting conditions among preschool children in rural Rwanda," Malaria Journal, vol. 14440 pages, 2015.

[44] J. Ndamukong-Nyanga, H. Kimbi, I. Sumbele et al., “A crosssectional study on the influence of altitude and urbanisation on Co-infection of malaria and soil-transmitted helminths in Fako division, south west Cameroon," International Journal of Tropical Disease \& Health, vol. 8, no. 4, pp. 150-164, 2015. 
[45] N. Sakwe, J. Bigoga, J. Ngondi et al., "Relationship between malaria, anaemia, nutritional and socio-economic status amongst under-ten children in the North region of Cameroon: a cross-sectional assessment," PLoS One, vol. 14, no. 6, 2019.

[46] D. I. Thurnham and G. P. McCabe, "Influence of infection and inflammation on biomarkers of nutritional status with an emphasis on vitamin A and iron," in Report: Priorities in the Assessment of Vitamin A and Iron Status in PopulationsWorld Health Organization, Geneva, Switzerland, 2012.

[47] J. F. Collins, M. Wessling-Resnick, and M. D. Knutson, "Hepcidin regulation of iron transport," The Journal of $\mathrm{Nu}$ trition, vol. 138, no. 11, pp. 2284-2288, 2008.

[48] J. T. Pinlap, "Anaemia in children under 5 years of age in Cameroon: a silent burden at the core of a rural-urban rivalry," Sight and Life, vol. 29, no. 2, 2015.

[49] V. Curtis and S. Cairncross, "Effect of washing hands with soap on diarrhoea risk in the community: a systematic review," The Lancet Infectious Diseases, vol. 3, no. 5, pp. 275-281, 2003.

[50] L. P. Leal, F. M. Batista, P. I. Lira, J. N. Figueiroa, and M. M. Osorio, “"Prevalence of anaemia and associated factors in children aged 6-59 months in Pernambuco, Northeastern Brazil," Brazilian Journal of Nutrition, vol. 91, pp. 307-15, 2011.

[51] C. R. K. Zuffo, M. M. Osório, C. A. Taconeli, S. T. Schmidt, B. H. Corrêa da Silva, and C. C. B. Almeida, "Prevalence and risk factors of anemia in children," Jornal de Pediatria (Versão em Português), vol. 92, no. 4, pp. 353-360, 2016.

[52] L. E. Thornton, J. R. Pearce, L. Macdonald, K. E. Lamb, and A. Ellaway, "Does the choice of neighbourhood supermarket access measure influence associations with individual-level fruit and vegetable intake? A case study from Glasgow," International Journal of Health Geography, vol. 11, no. 29, 2012.

[53] C. Y. Tata, A. Ickowitz, B. Powell, and E. K. Colecraft, "Dietary intake, forest foods, and anaemia in Southwest Cameroon," PLoS One, vol. 14, no. 4, Article ID e0215281, 2019.

[54] C. Termote, M. M. Bwama, D. B. Dhe'da, L. Huybregts et al., "A biodiverse rich environment does not contribute to a better diet: a study from DR Congo," PLoS One, vol. 7, no. 1, Article ID e30533, 2012.

[55] B. Powell, S. Thilsted, A. Ickowitz, C. Termote, T. Sunderland, and A. Herforth, "Improving diet with wild and cultivated biodiversity from across the landscape," Food Security, vol. 5, pp. 1-20, 2015.

[56] H. K. Kimbi, H. U. Ajeagah, F. C. Keka et al., "Asymptomatic malaria in school children and evaluation of the performance characteristics of the PartecCyscope ${ }^{\circledR}$ in the Mount Cameroon region," Journal of Bacteriology and Parasitology, vol. 3, no. 2, 2012.

[57] E. A. Okiro and R. W. Snow, "The relationship between reported fever and Plasmodium falciparum infection in African children," Malaria Journal, vol. 9, no. 99, 2010.

[58] I. Nkuo-Akenji, E. M. Sumbele, A. Njunda, M. Samje, and L. Kamga, "The burden of malaria and malnutrition among children less than 14 years of age in a rural village of Cameroon," African Journal of Food, Agriculture, Nutrition and Development, vol. 8, no. 3, pp. 252-264, 2008.

[59] M. S. Rahman, M. Mushfiquee, M. S. Masud, and T. Howlader, "Association between malnutrition and anaemia in under-five children and women of reproductive age: evidence from Bangladesh Demographic and Health Survey 2011,” PLos One, vol. 14, no. 7, Article ID e0219170, 2011.
[60] R. Khanam, H. S. Nghiem, and M. M. Rahman, "The impact of childhood malnutrition on schooling: evidence from Bangladesh," Journal of Biosocial Science, vol. 43, no. 4, pp. 437-451, 2011.

[61] WHO, Nutritional Anaemia: Report of a WHO scientific Group, World Health Organization, Geneva, Switzerland, 1968.

[62] S. Koka, M. Föller, G. Lamprecht et al., "Iron deficiency influences the course of malaria in Plasmodium berghei infected mice," Biochemical and Biophysical Research Communications, vol. 357, no. 3, pp. 608-614, 2007.

[63] D. Kejo, P. Petrucka, H. Martin, M. Kimanya, and T. Mosha, "Prevalence and predictors of anemia among children under 5 years of age in Arusha district, Tanzania," Pediatric Health, Medicine and Therapeutics, vol. 9, pp. 9-15, 2018.

[64] C. Nyaruhucha, J. Msuya, P. Mamiro, and A. Kerengi, "'Nutritional status and feeding practices of under-five children in Simanjiro district, Tanzania," Tanzania Journal of Health Resources, vol. 8, no. suppl 3, p. 162, 2006.

[65] Pava, F. H. Burdam, I. Handayuni et al., "Submicroscopic and asymptomatic Plasmodium parasitaemia associated with significant risk of anaemia in Papua, Indonesia," PLoS One, vol. 11, no. 10, Article ID 0165340, 2016.

[66] Y. L. Bahati, J. Delanghe, G. B. Balaluka, A. S. Kishabongo, and J. Philippe, "Asymptomatic sub-microscopic Plasmodium infection is highly prevalent and is associated with anemia in children younger than 5 years in South Kivu/democratic Republic of Congo," The American Journal of Tropical Medicine and Hygiene, vol. 102, no. suppl.5, pp. 1048-1055, 2020. 